\title{
Ciprofloxacin Sensitivity of Staphylococcus Strains Isolated at the Sylvanus Olympio University Hospital, Togo
}

\author{
Mounerou Salou1,2*, Degninou Yehadji ${ }^{3}$ Koumavi Ekouevi' ${ }^{2}$ Sika Dossim ${ }^{2,4}$, \\ Chantal Tsogou ${ }^{3}$, Yawo Tufa Nyasenu1,2, Fiali Lack ${ }^{1,2}$, Mireille Prince-David1,2, \\ Anoumou Y. Dagnra1,2 \\ ${ }^{1}$ Service de Microbiologie CHU Sylavanus Olympio, Lomé,Togo \\ ${ }^{2}$ Département des Sciences fondamentales de la Faculté des Sciences de la Santé ,Université de Lomé , \\ Lomé, Togo \\ ${ }^{3}$ Ecole Supérieure des Techniques Biologiques et Alimentaires, Université de Lomé, Lomé,Togo \\ ${ }^{4}$ Service laboratoire CHU Campus, Lomé, Togo \\ Email: ${ }^{*}$ mounerous@gmail.com
}

Received 27 October 2014; revised 28 November 2014; accepted 16 December 2014

Academic Editor: Ping Yang, Mayo Clinic, USA

Copyright @ 2014 by authors and Scientific Research Publishing Inc.

This work is licensed under the Creative Commons Attribution International License (CC BY).

http://creativecommons.org/licenses/by/4.0/

(c) (i) Open Access

\section{Abstract}

Background: Staphylococcal infections are managed by hygiene measures and usage of antibiotics. The first-line treatment refers to beta-lactamins. However, the emergence of beta-lactamin resistant staphylococcal strains has been reported, as demonstrated by a study conducted in Lomé University Teaching Hospital, Bacteriology Laboratory (2001) on 742 Staphylococcus aureus strains which revealed $67.00 \%$ of them where methicillin resistant. In this setting of emergent methicillin-resistant strains, the second-line treatments are prescribed by clinicians without antibiograms. Fluoroquinolones are the first preference molecules used for second-line treatment because of their efficacy and affordability. We want to contribute to setting monitoring and alertmaking tools for drug prescribers. Thus we conducted this study, aiming to determine the frequency of $S$. aureus and coagulase-negative staphylococci (CNS) strains isolation in different types of biologic samples, and to investigate the link between methicillin resistance and ciprofloxacin resistance. Methods: We conducted this study from January 2006 through Jun 2010. The Microbiology Laboratory Service collected and analyzed samples for diagnostic purpose from inpatients and outpatients consulted in the hospital. We collected and analyzed de-identified data on these patients to form laboratory records. Bacteriological analyses in which ciprofloxacin have not been

\footnotetext{
"Corresponding author.
}

How to cite this paper: Salou, M., Yehadji, D., Ekouevi, K., Dossim, S., Tsogou, C., Nyasenu, Y.T., Lack, F., Prince-David, M. and Dagnra, A.Y. (2014) Ciprofloxacin Sensitivity of Staphylococcus Strains Isolated at the Sylvanus Olympio University Hospital, Togo. Pharmacology \& Pharmacy, 5, 1143-1147. http://dx.doi.org/10.4236/pp.2014.513124 
tested were excluded. Results: Over the 1108 staphylococal strains isolated from various biological samples processed, 751 were Staphylococcus aureus and 357 were coagulase-negative staphylococci. The strains sensitivity profile is for all isolates. The majority of germs were $S$. aureus and $20 \%$ of them were ciprofloxacin-resistant. The probability of a patient who has an infection caused by $S$. aureus increases his stay in high-risk settings such as intensive care unit, surgical intervention, extended hospitalization, use of a catheter. The use of broad-spectrum antibiotics increases the risk of multidrug-resistant strains. Conclusion: This study highlights the recurring issue of over consumption of antibiotics in nowadays medical treatments. There is a need to raise awareness about the rational use of antibiotics in general and fluoroquinolones particularly.

\section{Keywords}

\section{Staphylococcus, Ciprofloxacin, Methicillin, Resistance}

\section{Introduction}

Staphylococci are ubiquitous germs part of the human and animal cutaneous-mucous flora. They can be spread by a healthy carrier. In all likelihood, they are secondary disseminated in the environment by squama and remain persistent because of their resistance to desiccation temperature variations and osmotic chock. When cutaneousmucous barriers are damaged or when the host's defenses are diminished, theses germs induce cutaneous, bone, and visceral infections, even worse, they can lead to septicemia [1].

When hygiene measures are not respected and patients are note isolated in hospitals, staphylococcus infections can lead to hospital epidemics. Staphylococcus can also persist endemically and lead to a high rate of nosocomial infections. The seriousness of these infections is linked to symptoms severity and to the therapeutic difficulties because of the multi resistance to antibiotics [1] [2].

The management of infections requires hygiene measures and use of antibiotics. Beta-lactamins are used as first-line treatment. However, the emergence of beta-lactamin resistant staphylococcal strains has been reported. A study conducted at the Sylvanus Olympio University Hospital (CHU-Tokoin, name of the hospital at the time of that study), Laboratory of Bacteriology (2001) on 742 Staphylococcus aureus strains revealed that $67.00 \%$ of them where methicillin resistant (Methi-R) [3].

In the setting of emerging methicillin-resistant strains, second-line treatments are prescribed. These treatments are prescribed in our health services by clinicians without antibiogram evidences. The first preference molecules used for this purpose are fluoroquinolones because of their efficacy (good diffusion in adipose tissue) and affordability [4]. We conducted this study to assess ciprofloxacin resistance (Cipro-R) of staphylococcal isolates in our settings.

\section{Materials and Method}

Study design: We conducted a cross sectional study covering the period of January 2006 through June 2010. We collected disidentified data from the CHU-SO (precedently named CHU Tokoin), Bacteriology Laboratory records. These records are results of samples (vaginal swab, urethral swab, coproculture, urine, hemoculture, pus and antibiograms) from inpatients and outpatients consulted in the hospital, and were processed in the laboratory for diagnostic purpose only. Results of bacteriological tests for which ciprofloxacin was not tested were excluded.

Laboratory method: Methicillin-resistance and phenotypes of staphylococci antibiotics resistance is analyzed with agar diffusion method. The Mueller-Hinton agar is used for the antibiogram. Each S. aureus or CNS colony isolated is seeded in $4 \mathrm{ml}$ sterile peptone-water contained in hemolysis tube. After 24 hours at $37^{\circ} \mathrm{C}$ incubation in darkness, 2 or 3 drops of the broth media is added with a Pasteur pipette to $10 \mathrm{~mL}$ sterile distilled water contained in a tube with screw cap. The inoculum prepared is diluted until obtaining a turbidity visually equivalent to 0.5 McFarland [5].

Petri dishes are dried $15 \mathrm{~min}$ in a sterilizer. Dried dishes are seeded by inoculation, and the inoculum surplus is sucked up from the surface of the agar medium with a Pasteur pipette carried with a pear. A disk of each anti- 
biotic is dispensed with a sterile plier: penicillin $(\mathrm{P})$, kanamycin $(\mathrm{K})$, tobramycin $(\mathrm{T})$, gentamycin $(\mathrm{G})$, lincomycin (L), erythromycin (E), pristinamycin (PT), ofloxacin (OFX), pefloxacin (PEF) or ciprofloxacin (CIP), vancomycin (V), oxacillin (OX) and cefoxitin (FOX). Oxacillin and cefoxitin are used to determine methicillin-resistance [6]. Oxacillin discs are tested on Muller-Hinton media added with $5 \% \mathrm{NaCl}$ and dropped alone at the middle of the Petri plastic dish measuring $50 \mathrm{~mm}$ diameter. The other disks are dispensed by maximum of six in a Petri plastic dish measuring $90 \mathrm{~mm}$ diameter. The erythromycin disk is carefully dropped at $2 \mathrm{~cm}$ of the lincomycin disk. A pre-diffusion of antibiotics is carried out for $15 \mathrm{~min}$ at laboratory room temperature before incubation of dishes in a dark sterilizer at $37^{\circ} \mathrm{C}$ for 18 to 24 hours without repetition.

Interpretation of the antibiogram is carried out following the guidelines of The French Society of Microbiology. Inhibition area diameters are measured with a ruler at $0.5 \mathrm{~mm}$ approximation [7]. Ciprofloxacin disc charge was $5 \mu$ g. Staphylococcus spp strains Cipro-R were those with inhibition diameter $<22 \mathrm{~mm}$. Oxacillin disc and cefoxitin disc charge was respectively $5 \mu \mathrm{g}$ and $30 \mu \mathrm{g}$. Staphylococcus spp strains were classified Methicllin-resistant if inhibition diameter was $<20 \mathrm{~mm}$ or $<25 \mathrm{~mm}$ respectively for oxacillin and cefoxitin.

Data analysis: The data were collected and processed with Epi Info Version 6.04 and Microsoft ${ }^{\circledR}$ Excel 2007 software. We compared values (Chi-square test and relative risk) at 0.05 significance level.

\section{Results}

Over a total of 1108 staphylococcus strains isolated during the study period, 751 (68\%) were S. aureus and 357 (32\%) were coagulase-negative staphylococci (CNS).The majority (53\%) of staphylococci strains were isolated from inpatients versus outpatients (43\%). With regard to biological samples, almost all staphylococci strains (82\% of $S$. aureus and $72.98 \%$ of CNS) were isolated from pus samples (Table 1 ).

$85.98 \%$ of Staphylococcus aureus strains isolated were ciprofloxacin sensitive (S), and $69.55 \%$ of CNS strains were ciprofloxacin sensitive. During the same period, the prevalence of ciprofloxacin resistant staphylococci strains increased significantly: $\mathrm{p}<0.01$ (Figure 1). The overall methicillin-resistance is $37.38 \%$.

The relative risk (RR) of ciprofloxacin-resistant staphylococci of being methicillin-resistant was 1.85 and it was statistically significant ( $<<0.01$ ). The RR of ciprofloxacin-resistant CNS of being methicillin-resistant was 1.86 and it was statistically significant $(\mathrm{p}<0.01)$ (Table 2). The RR of methicillin-resistant staphylococci of being ciprofloxacin-resistant was 2.54 and it was statistically significant $(\mathrm{p}<0.01)$. The RR of methicillin-resistant CNS of being ciprofloxacin-resistant was 2.87 and it was statistically significant $(\mathrm{p}<0.01)$ (Table 3$)$.

\section{Discussions}

Upon this study, we found that there are more staphylococci strains isolated from inpatients than from outpatients settings. Several studies show that methicillin-resistant Staphylococcus aureus (MRSA) are being important bacterial pathogens in hospital setting, but our result (37.38\%) is much higher: 6.64\% in the United States (2010), 5.97\% in Canada (1999) and 0.8\% in Japan (2003) [8]-[10]. This could be explained by the emergence of resistant strains and increasing hospital acquired infections resulting from lack of good care practices and infections control guidelines.

Isolates originated from various samples. Pus and miscellaneous punctures were the most represented, followed by urine and hemoculture or blood culture. S. aureus was the most isolated from pus and miscellaneous punctures (83.62\%) as compared to CNS strains (72.98\%). As opposed to pus samples and miscellaneous punctures, there were more CNS strains (11.42\%) than S. aureus (4.26\%) isolated from hemocultures. This could be related to probable samples contamination during blood collection. This distribution is similar to the findings of Akoua-Koffi et al. (2004) in Côte d'Ivoire (64.7\% from pus versus 9.4\% isolated urine and other samples) and could be linked to the pathogenicity of staphylococcus strains according to the colonization site [11].

Our findings demonstrated an increasing rate of ciprofloxacin resistance. This could be induced by antibiotherapy practices. For example, self-medication of the population in the treatment of unproved typhoid and paratyphoid fevers, in view of the drug affordability and the various generic-type packaging of the molecule.

This ciprofloxacin-resistance is correlated with an increased methicillin-resistance as fluoroquinolone-resistance staphylococci have a methicillin-resistance profile. We found that ciprofloxacin-resistant $S$. aureus are 1.85 -fold at higher risk of being methicillin-resistant. Methicillin-resistance is also correlated with an increased ciprofloxacin-resistance. Methicillin-resistant S. aureus are 2.54-fold at higher risk of being ciprofloxacin-resistant, meaning that failure of the first line treatment increases the risk of the second line treatment failure. 
Table 1. Distribution of staphylococci by type of biological samples.

\begin{tabular}{cccc}
\hline Type of sample & S. aureus $(\%)$ & S. CN $(\%)$ & Total \\
\hline Pus & $628(83,62)$ & $262(73,39)$ & 890 \\
Urine & $49(6,52)$ & $46(12,88)$ & 95 \\
Blood culture & $32(4,26)$ & $41(11,48)$ & 73 \\
Vaginal swabs & $23(3,06)$ & $2(0,56)$ & 25 \\
Sperm & $17(2,26)$ & $3(0,84)$ & 20 \\
Urethral scraping & $2(0,26)$ & $3(0,84)$ & 5 \\
Total & $751(100)$ & $357(100)$ & 1108 \\
\hline
\end{tabular}

Table 2. Methicillin antibiogram profile of staphylococci strains according to their ciprofloxacin antibiogram profile.

\begin{tabular}{ccccc}
\hline & Methi-R & Methi-S & RR & P-value \\
\hline Cipro-R S. aureus & 112 & 74 & 1.84 & $<0.01$ \\
Cipro-S S. aureus & 291 & 601 & & \\
Cipro-R CNS & 73 & 26 & 1.86 & $<0.01$ \\
Cipro-S CNS & 98 & 149 & & \\
\hline
\end{tabular}

Table 3. Ciprofloxacin antibiogram profile of staphylococci strains according to their methicillin antibiogram profile.

\begin{tabular}{ccccc}
\hline & Cipro-R S. aureus & Cipro-S S. aureus & RR & P-value \\
\hline Methi-R & 112 & 291 & 2.54 & $<0.01$ \\
Methi-S & 74 & 601 & & \\
\hline & Cipro-R CNS & Cipro-S CNS & RR & P-value \\
\hline Methi-R & 73 & 98 & 2.87 & $<0.01$ \\
Methi-S & 26 & 149 & & \\
\hline
\end{tabular}

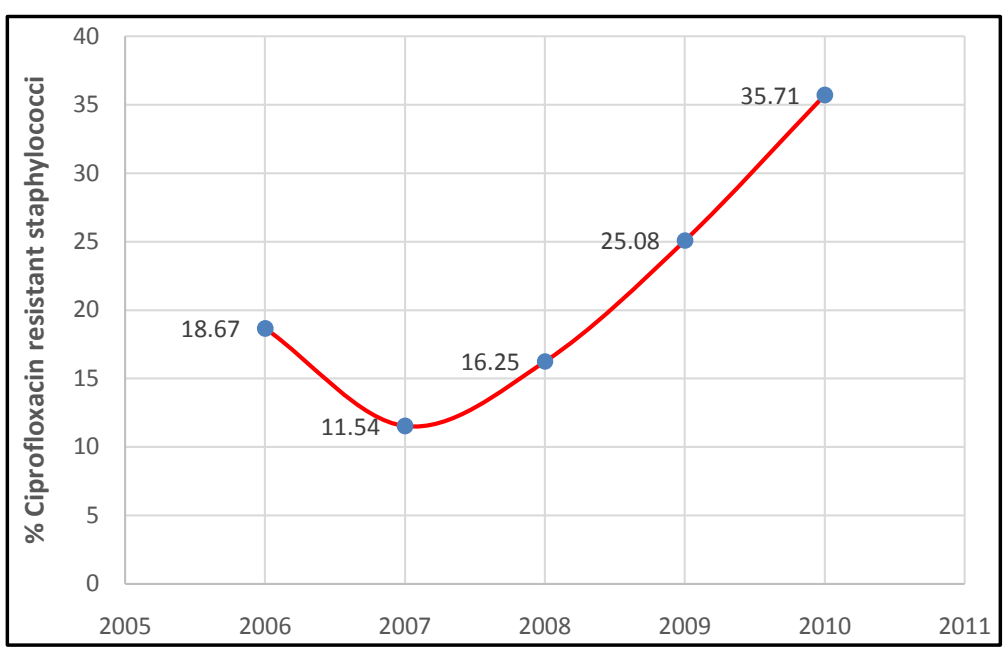

Figure 1. Prevalence and trends of ciprofloxacin resistant staphylococci strains. 


\section{Conclusion}

This study highlights the recurring issue of over consumption of antibiotics in nowadays medical treatments. For better treatment outcomes, there is a need to raise awareness about the rational and well-targeted use of antibiotics in general and fluoroquinolones specifically. We recommend that, for patients displaying failure of the probabilistic in the first-line antibiotherapy (fluoroquinolones or betalactamins), the second-line treatment is considered on the basis of the antibiogram.

\section{References}

[1] Mylotte, J.M., McDermott, C. and Spooner, J.A. (1987) Prospective Study of 114 Consecutive Episodes of Staphylococcus aureus Bacteremia. Clinical Infectious Diseases, 9, 891-907. http://dx.doi.org/10.1093/clinids/9.5.891

[2] Boyce, J.M., White, R.L. and Spruill, E.Y. (1983) Impact of Methicillin-Resistant Staphylococcus aureus on the Incidence of Nosocomial Staphylococcal Infections. The Journal of Infectious Diseases, 148, 763. http://dx.doi.org/10.1093/infdis/148.4.763

[3] Dagnra, A.Y., Hounkpati, A. and Prince-David, M. (2001) Fort Pourcentage de souches de Staphylococcus aureus résistantes à la méticilline au CHU de Lomé (Togo). Médecineet et Maladies Infectieuses, 31, 14-18.

[4] Wolfson, J.S. and Hooper, D.C. (1989) Fluoroquinolone Antimicrobial Agents. Clinical Microbiology Reviews, 2, 378424.

[5] National Committee for Clinical Laboratory Standards (2003) Methods for Dilution Antimicrobial Susceptibility Tests for Bacteria that Grow Aerobically: Approved Standard M7-A6. NCCLS, Wayne, Pennsylvania, USA.

[6] Datta, P., et al. (2011) Evaluation of Various Methods for the Detection of Methicillin-Resistant Staphylococcus aureus Strains and Susceptibility Patterns. Journal of Medical Microbiology, 60, 1613-1616. http://dx.doi.org/10.1099/jmm.0.032219-0

[7] Bonnet, R., et al. (2011) Comité de l’antibiogramme de la Société Française de Microbiologie: Recommandations 2011. Société Française de Microbiologie, Paris.

[8] Jarvis, W.R., Jarvis, A.A. and Chinn, R.Y. (2012) National Prevalence of Methicillin-Resistant Staphylococcus aureus in Inpatients at United States Health Care Facilities, 2010. American Journal of Infection Control, 40, 194-200. http://dx.doi.org/10.1016/j.ajic.2012.02.001

[9] Simor, A.E., et al. (2001) The Evolution of Methicillin-Resistant Staphylococcus aureus in Canadian Hospitals: 5 Years of National Surveillance. Canadian Medical Association Journal, 165, 21-26.

[10] Kobayashi, H. (2005) National Hospital Infection Surveillance on Methicillin-Resistant Staphylococcus aureus. Journal of Hospital Infection, 60, 172-175. http://dx.doi.org/10.1016/j.jhin.2004.12.010

[11] Akoua-Koffi, C., et al. (2004) La méticillino-résistance de Staphylococcus aureus isolés à Abidjan (1998-2001): Un nouveau problème en milieu hospitalier. Medecineet et Maladies Infectieuses, 34, 132-136.

http://dx.doi.org/10.1016/j.medmal.2003.12.001 
Scientific Research Publishing (SCIRP) is one of the largest Open Access journal publishers. It is currently publishing more than 200 open access, online, peer-reviewed journals covering a wide range of academic disciplines. SCIRP serves the worldwide academic communities and contributes to the progress and application of science with its publication.

Other selected journals from SCIRP are listed as below. Submit your manuscript to us via either submit@scirp.org or Online Submission Portal.
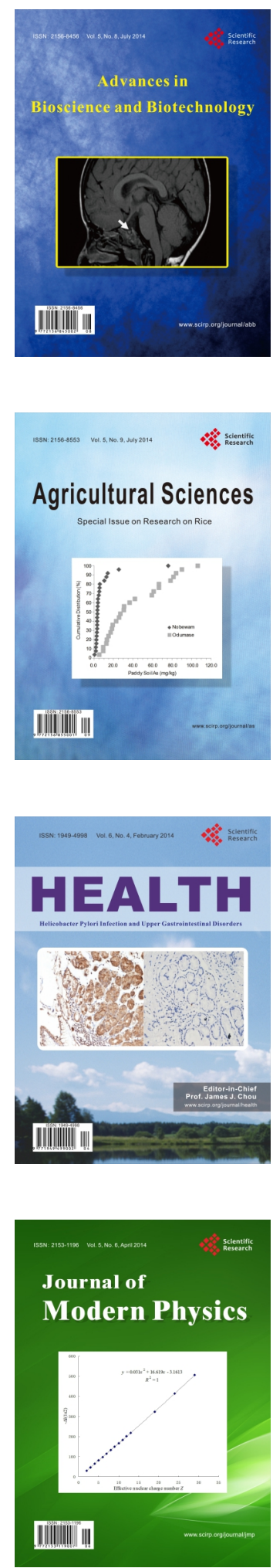
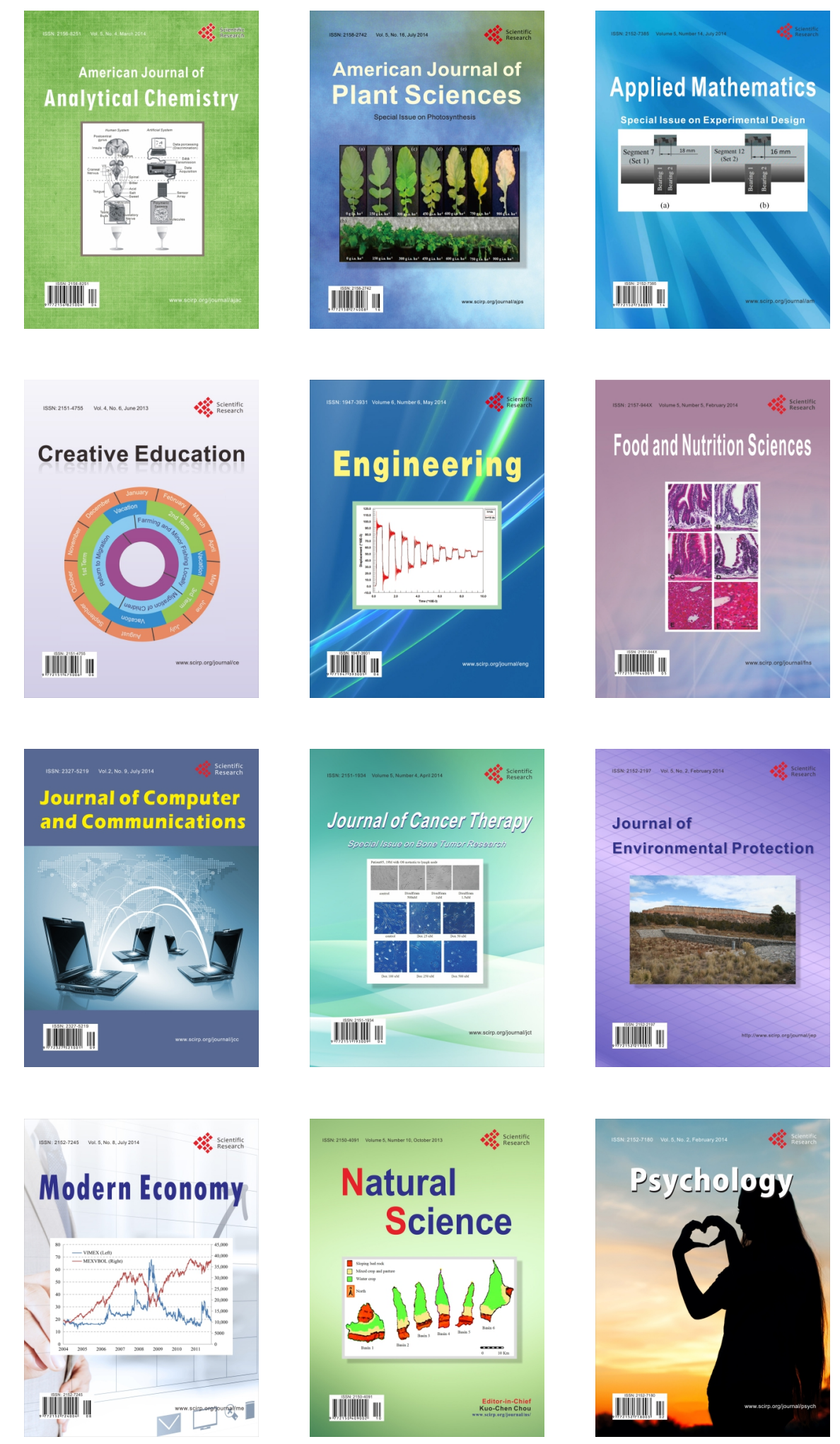